\title{
Relationship between Fat Mass and Serum High-Sensitivity C-Reactive Protein Levels in Prevalent Hemodialysis Patients
}

\author{
Eiji Ishimura $^{a}$ Senji Okuno ${ }^{c}$ Naoki Tsuboniwa ${ }^{c}$ Shigeichi Shojic \\ Tomoyuki Yamakawa $^{c}$ Yoshiki Nishizawa $^{\mathrm{b}}$ Masaaki Inaba $^{\mathrm{b}}$ \\ Departments of a Nephrology and ${ }^{b}$ Metabolism, Endocrinology, and Molecular Medicine, Osaka City University \\ Graduate School of Medicine, and 'Shirasagi Hospital, Osaka, Japan
}

\section{Key Words}

Fat mass $\cdot$ Lean mass $\cdot$ Truncal fat mass $\cdot$ C-reactive protein $\cdot$ Dual X-ray absorptiometry $\cdot$ Hemodialysis

\begin{abstract}
Background/Aims: Little is known about the relationship between fat mass distribution and chronic inflammation in dialysis patients, in whom chronic inflammation is related to morbidity and mortality. Methods: The fat and lean masses (truncal and nontruncal) of 452 hemodialysis patients (age: $64 \pm 11$ years; hemodialysis duration: $89 \pm 77$ months; $37 \%$ diabetics) were measured by dual X-ray absorptiometry and their association with high-sensitivity C-reactive protein (hsCRP) was examined. Results: The fat mass of the high hsCRP group ( $\mathrm{n}=106$ ) was significantly higher than that of the normal hsCRP group ( $n=346 ; p<0.05)$; there were no significant differences in lean mass between the two groups. Truncal fat mass of the former group was significantly greater than that of the latter $(p<0.05)$, but there was no significant difference in nontruncal fat mass between the two groups. In multiple regression analysis, truncal fat mass ( $\beta=$ $0.227, p<0.01$ ) was significantly and independently associated with serum hsCRP levels after adjustment for age, gender and serum albumin $\left(R^{2}=0.137, p<0.01\right)$, whereas
\end{abstract}

nontruncal fat mass was not. Conclusion: Fat mass, particularly truncal fat mass, but not lean body mass, was significantly associated with serum hsCRP levels. The results suggest that truncal fat mass exhibits a distinct effect on chronic inflammation in hemodialysis patients.

Copyright $\odot 2011$ S. Karger AG, Basel

\section{Introduction}

In the general population, obesity, particularly increases in visceral fat, is characterized by chronic lowgrade inflammation with increased serum inflammatory cytokines and is considered to be a risk factor for atherosclerosis, cardiovascular disease and increased mortality $[1,2]$. Adipose tissue is strongly associated with these cytokine abnormalities [1-3]. Inflammatory cytokines, such as interleukin 6 (IL-6), stimulate the production of hepatic C-reactive protein (CRP) $[1,4]$. A significant role of visceral fat in obese patients is particularly emphasized in the increase in inflammatory cytokines, leading to increases in CRP $[2,3,5,6]$.

In hemodialysis patients, chronic inflammation, as represented by increased IL- 6 and CRP, is strongly associated with morbidity and mortality [7-9]. However, little

\section{KARGER}

Fax +41613061234 E-Mail karger@karger.ch www.karger.com
Eiji Ishimura, MD

Department of Nephrology, Osaka City University Graduate School of Medicine 1-4-3 Asahi-machi, Abeno-ku

Osaka 545-8585 (Japan)

Tel. +81 66645 3806, E-Mail ish@med.osaka-cu.ac.jp 
is known about the association between chronic inflammation and body composition, such as body fat mass, in hemodialysis patients. Only a few studies examining a relatively small number of patients have reported any relationship between abdominal fat and low-grade inflammation in patients with end-stage renal disease [10-12]. The purpose of the present study was to investigate the relationship between serum high-sensitivity CRP (hsCRP) and fat mass in a large number of prevalent hemodialysis patients, and in particular to investigate which fat mass, truncal or nontruncal (the former of which represents visceral fat mass [11]), is more associated with chronic inflammation.

\section{Patients and Methods}

\section{Patients}

A total of 452 stable maintenance hemodialysis patients (286 males and 166 females) at Shirasagi Hospital, Osaka, Japan, with hemodialysis duration of more than 3 months were examined in the present study. Patients with malignancy and clinically overt infection were excluded. The mean $( \pm S D)$ age was 64 years $( \pm 11)$ and the median (range) of hemodialysis duration was 66.7 months (3.0-392.7). There were 169 diabetics and 283 nondiabetics. In all patients, 4-hour hemodialysis was performed three times a week with bicarbonate dialysate. All patients provided informed consent prior to participation in the present study. This study was approved by the ethics review committee of the hospital.

Blood was collected before each hemodialysis session. Blood urea nitrogen, serum creatinine, albumin, calcium, phosphate and hemoglobin were measured by routine laboratory methods. hsCRP was measured by turbid immunoassay. Patients with hsCRP $>3 \mathrm{mg} / \mathrm{dl}$ were excluded because of possible acute or chronic infection.

All patients were divided into two groups according to their serum hsCRP levels: normal hsCRP $(\mathrm{n}=346)$ with hsCRP $<0.3$ $\mathrm{mg} / \mathrm{dl}$ (i.e. normal levels of hsCRP) and high hsCRP $(\mathrm{n}=106)$ with hsCRP $\geq 0.3 \mathrm{mg} / \mathrm{dl}$.

\section{Measurement of Fat Mass by Dual X-Ray Absorptiometry}

At Shirasagi Hospital, measurements of bone mineral density are performed routinely in all patients: annually, near the birthday of each patient, by dual X-ray absorptiometry (DXA; QDR4500A; Hologic, Waltham, Mass., USA) and 21-24 h after completion of a dialysis session. When bone mineral density measurements were performed by DXA, fat mass and lean mass of the patients were also measured. In the measurements, the fat mass and lean mass of 5 parts of the body were measured separately (fig. 1) as reported previously [11]: the trunk, left and right upper extremities, and left and right lower extremities. Nontruncal fat mass and lean mass were calculated by summing up the fat mass and lean mass of the four extremities, respectively, in each patient. The percent truncal fat mass was calculated by division of the truncal fat mass by the total fat mass and expressed as a percentage.

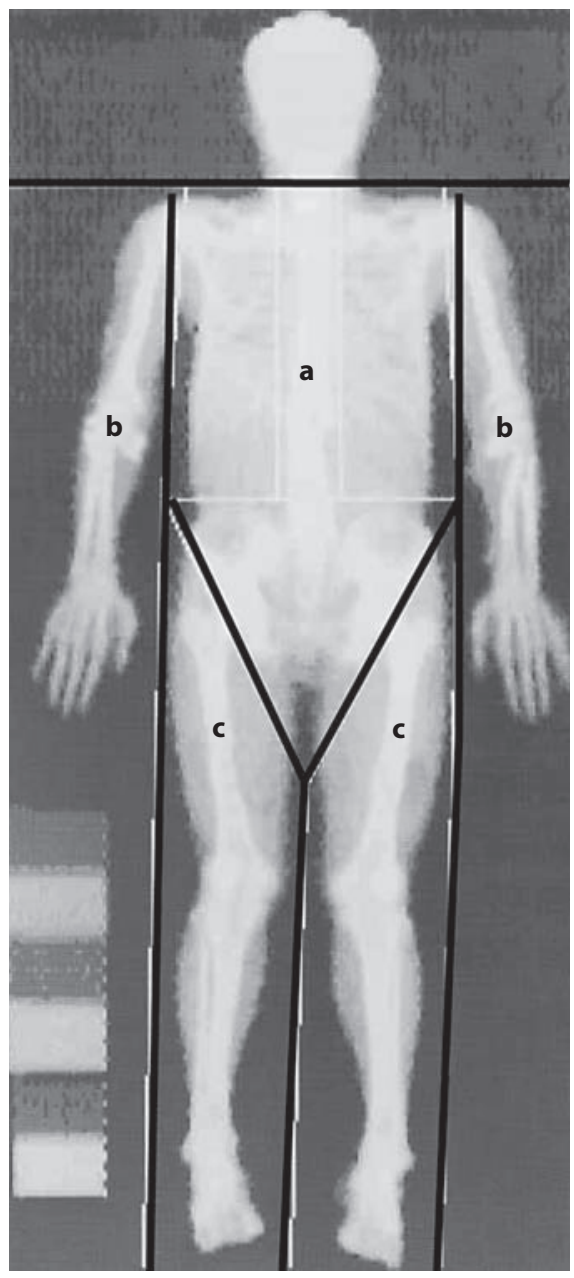

Fig. 1. DXA measurement of 5 parts of the body. Fat mass and lean mass of 5 parts of the body were measured separately, as previously reported [11]: trunk (a), upper extremities (b), and lower extremities (c).

\section{Statistical Analysis}

Data are presented as the mean \pm SD. An unpaired Student t test was performed to compare the values between the two groups. Data of skewed deviation (hemodialysis duration) were compared by a Mann-Whitney U test. Categorical values were compared by a $\chi^{2}$ test between the two groups. Correlation and linear regression analyses were performed to examine the relationship between DXA parameters and serum hsCRP; serum hsCRP was transformed logarithmically because of its skewed distribution. Multiple regression analysis was performed to examine the independent association of several parameters with the $\log$ hsCRP. In the analysis, gender and diabetes were entered as a dummy variable (male 0 , female 1 ; non-diabetes 0 , diabetes 1 ). $\mathrm{p}<0.05$ was considered significant. All analyses were performed using StatView 5 (SAS Institute Inc., Cary, N.C., USA) designed for a Windows personal computer.
Ishimura/Okuno/Tsuboniwa/Shoji/ Yamakawa/Nishizawa/Inaba 


\section{Results}

Table 1 shows the clinical characteristics of the patients, according to normal $(\mathrm{n}=346)$ and high hsCRP $(\mathrm{n}=106)$. There were no significant differences in age, gender, hemodialysis duration, body weight or prevalence of diabetes, calcium, phosphate and hemoglobin between the two hsCRP groups. Serum albumin and blood urea nitrogen were significantly lower in the high hsCRP group compared with the normal hsCRP group $(\mathrm{p}<0.01$ and $\mathrm{p}<0.05$, respectively).

Table 1 shows the DXA measurements of the total fat mass, total lean mass, truncal fat mass, nontruncal fat mass and percent truncal fat mass. The total fat mass of the high hsCRP group was significantly greater than that of the normal hsCRP group $(14.80 \pm 6.64$ vs. $13.40 \pm$ $5.44 \mathrm{~kg}, \mathrm{p}<0.05)$, but there were no significant differences in the total lean mass between the two groups. While there was no significant difference in the nontruncal fat mass between the two groups, the truncal fat mass of the high hsCRP group was significantly greater than that of the normal hsCRP group (7.69 \pm 4.29 vs. $6.67 \pm$ $3.38 \mathrm{~kg}, \mathrm{p}<0.05)$. The percent truncal fat mass out of the total fat mass was significantly greater in the high hsCRP group compared with the normal hsCRP group (49.7 \pm 8.0 vs. $47.8 \pm 8.7 \%, \mathrm{p}<0.05)$.

In all patients, there was a significant positive correlation between serum hsCRP and total fat mass ( $\mathrm{r}=0.186$, $p<0.0001$ ), but there was none between serum hsCRP and lean mass (fig. 2). There were significant positive correlations between the serum hsCRP and truncal fat mass $(\mathrm{r}=0.202, \mathrm{p}<0.0001$; fig. 2$)$, and between the serum hsCRP and nontruncal fat mass $(r=0.136, p<0.0001$; fig. 3), but there was no significant correlation between serum hsCRP and truncal lean mass, or between serum hsCRP and nontruncal lean mass (fig. 3, 4).

In multiple regression analysis, truncal fat mass $(\beta=$ $0.227, \mathrm{p}<0.01$ ) was significantly and independently associated with serum hsCRP in addition to other significant and independent variables of age, gender and serum albumin $\left(\mathrm{R}^{2}=0.137, \mathrm{p}<0.01\right)$; nontruncal fat mass was not a significant variable (table 2 ).

\section{Discussion}

In the present study, we demonstrated that fat mass, particularly truncal fat mass, but not lean body mass or nontruncal fat mass, was significantly greater in hemodialysis patients with higher hsCRP than in those with nor-
Table 1. Clinical characteristics of patients according to normal and high hsCRP

\begin{tabular}{|c|c|c|c|}
\hline & \multicolumn{2}{|l|}{ Patients with } & \multirow{2}{*}{$\begin{array}{l}\mathrm{p} \\
\text { value }\end{array}$} \\
\hline & $\begin{array}{l}\text { normal hsCRP } \\
(\mathrm{n}=346)\end{array}$ & $\begin{array}{l}\text { high hsCRP } \\
(\mathrm{n}=106)\end{array}$ & \\
\hline Age, years & $63.2 \pm 11.3$ & $65.6 \pm 11.6$ & 0.058 \\
\hline Gender, male/female & $215 / 131$ & $71 / 35$ & 0.430 \\
\hline \multicolumn{4}{|c|}{ Hemodialysis duration, months } \\
\hline Median & 71.1 & 59.2 & 0.071 \\
\hline Range & $3.0-392.7$ & $3.0-348.1$ & \\
\hline Body weight, kg & $54.3 \pm 10.8$ & $55.8 \pm 12.4$ & 0.233 \\
\hline Diabetes, yes/no & $125 / 221$ & $44 / 62$ & 0.375 \\
\hline Albumin, g/dl & $3.6 \pm 0.3$ & $3.4 \pm 0.4$ & $<0.001$ \\
\hline Blood urea nitrogen, $\mathrm{mg} / \mathrm{dl}$ & $69 \pm 14$ & $66 \pm 15$ & 0.027 \\
\hline Creatinine, $\mathrm{mg} / \mathrm{dl}$ & $11.1 \pm 2.6$ & $10.7 \pm 2.5$ & 0.146 \\
\hline Calcium, mg/dl & $9.7 \pm 0.9$ & $9.6 \pm 0.8$ & 0.157 \\
\hline Phosphate, mg/dl & $5.6 \pm 1.3$ & $5.6 \pm 1.6$ & 0.717 \\
\hline Hemoglobin, g/dl & $10.4 \pm 1.1$ & $10.2 \pm 1.5$ & 0.175 \\
\hline Total fat mass, kg & $13.40 \pm 5.44$ & $14.80 \pm 6.64$ & 0.028 \\
\hline Total lean mass, $\mathrm{kg}$ & $40.02 \pm 8.65$ & $39.54 \pm 8.49$ & 0.615 \\
\hline Truncal fat mass, $\mathrm{kg}$ & $6.67 \pm 3.38$ & $7.69 \pm 4.29$ & 0.011 \\
\hline Nontruncal fat mass, $\mathrm{kg}$ & $5.80 \pm 2.34$ & $6.15 \pm 2.48$ & 0.179 \\
\hline Percent truncal fat mass, $\%$ & $47.8 \pm 8.7$ & $49.7 \pm 8.0$ & 0.041 \\
\hline
\end{tabular}

Table 2. Factors affecting serum hsCRP levels (multiple regression analysis)

\begin{tabular}{lrc}
\hline & $\beta$ & p value \\
\hline Age (per 1 year) & 0.102 & 0.034 \\
Gender (male 0, female 1) & 0.152 & 0.001 \\
Hemodialysis duration (months) & 0.018 & 0.693 \\
Diabetes (absent 0, present 1) & -0.048 & 0.312 \\
Albumin (per 1 g/dl) & -0.278 & $<0.001$ \\
Truncal fat mass (per 1 kg) & 0.227 & 0.003 \\
Nontruncal fat mass (per 1 kg) & -0.007 & 0.926 \\
\hline $\mathrm{R}^{2}$ & 0.137 & $<0.001$ \\
\hline
\end{tabular}

mal hsCRP. Fat mass, both truncal and nontruncal, was significantly associated with hsCRP, but lean mass was not. Further, truncal fat mass was significantly and independently associated with hsCRP after adjustment for several confounders, but nontruncal fat mass was not. These results suggest that fat mass, particularly truncal fat mass, exhibit a distinct effect on low-grade inflammation in hemodialysis patients. 
Fig. 2. Relationship between serum hsCRP, total fat mass and lean mass. There was a significant positive correlation between serum hsCRP and total fat mass $(r=0.186$, $p<0.0001)$, but not between serum hsCRP and lean mass.

Fig. 3. Relationship between serum hsCRP, truncal fat mass and truncal lean mass. There was a significant positive correlation between serum hsCRP and truncal fat mass $(r=0.202, p<0.0001)$, but no significant correlation between serum hsCRP and truncal lean mass.

Fig. 4. Relationship between serum hsCRP, nontruncal fat mass and nontruncal lean mass. There was a significant positive correlation between serum hsCRP and nontruncal fat mass $(\mathrm{r}=0.136, \mathrm{p}<0.0001)$, but no significant correlation between serum hsCRP and nontruncal lean mass.
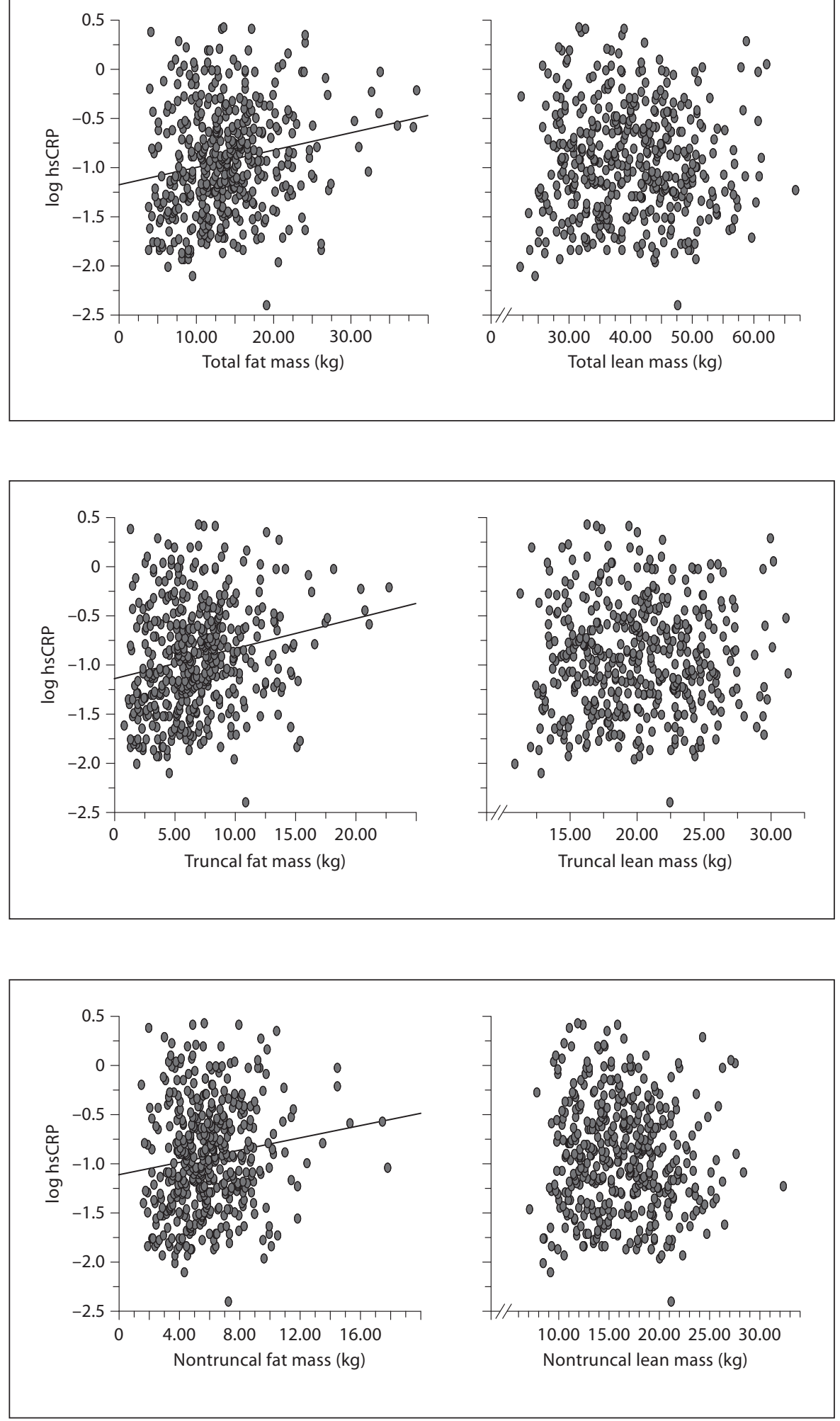

Ishimura/Okuno/Tsuboniwa/Shoji/ Yamakawa/Nishizawa/Inaba 
Several studies have examined the relationship between inflammatory cytokines and adipose tissue in the general population, a healthy adolescent population and obese patients $[2,3,5]$. In these studies, a significant role of adipose tissue, which is a rich source of many immune-related mediators such as IL-6, tumor necrosis factor- $\alpha$ (TNF- $\alpha)$ and adiponectin $[1,13]$, has been emphasized in the development of a systemic inflammatory state, leading to hepatic synthesis of acute phase inflammatory proteins such as CRP $[1,14]$. A significant role of visceral fat has been particularly emphasized in the increase in inflammatory cytokines and CRP $[2,5$, 6]. Thus, abdominal or central obesity with increased visceral fat has been considered to be a risk factor for increased systemic low-grade inflammation and risk for atherosclerosis and cardiovascular disease [1]. However, in patients with end-stage renal disease or who are undergoing hemodialysis, there have been only a few studies that have examined the relationship between lowgrade inflammation and visceral fat mass [10-12]. Kaysen et al. [12] examined visceral adipose tissue by magnetic resonance imaging in 48 patients with prevalent hemodialysis, and showed that the acute phase inflammatory protein, ceruloplasmin, was strongly associated with visceral adipose tissue. Gohda et al. [10] examined visceral fat area by computed tomography in 80 patients with prevalent hemodialysis, and showed that visceral fat area was a predominant determinant of CRP. Axelsson et al. [11] examined truncal and nontruncal fat mass with DXA (similar to our report) in 197 patients with end-stage renal disease shortly before the beginning of dialysis therapy. In their study on nondialyzed patients, lean body mass was not significantly associated with CRP, but total body fat mass and truncal fat mass were significantly associated with CRP. Although the present study examined patients with prevalent hemodialysis ( $n=452$, hemodialysis duration of $89 \pm 77$ months), the results of our study are consistent with their study. In the present study, although both truncal fat mass and nontruncal fat mass were significantly associated with hsCRP, truncal fat mass was significantly and independently associated with hsCRP after adjustment for several factors, including diabetes and nontruncal fat mass. Taken together with the previous studies, it is indicated that the visceral or truncal fat mass is an important contributor to increased chronic inflammation.

In dialysis patients, reverse epidemiology studies have emphasized that the mortality of obese patients is lower than that of nonobese patients $[15,16]$. In the previous studies [10-12] and our own, visceral or truncal fat is associated with increased low-grade inflammation as described above. In dialysis patients, chronic inflammation has been reported to be a significant predictor of cardiovascular disease and mortality [7-9, 17]. Previous studies [10-12] and our own are cross-sectional in design. Clearly, long-term prospective studies are needed to evaluate whether visceral or truncal fat mass is associated with cardiovascular disease and mortality.

In conclusion, the results of the present study showed that fat mass, but not lean body mass, was significantly associated with serum hsCRP. Truncal fat mass, but not nontruncal fat mass, was significantly and independently associated with serum hsCRP, suggesting that truncal fat mass exhibits a distinct effect on low-grade inflammation in hemodialysis patients and is possibly associated with morbidity and mortality, although further studies are needed to clarify the prognostic effect of abdominal obesity in dialysis patients.

\section{References}

$>1$ Berg AH, Scherer PE: Adipose tissue, inflammation, and cardiovascular disease. Circ Res 2005;96:939-949.

$\checkmark 2$ Nishida M, Moriyama T, Sugita Y, Yamauchi-Takihara K: Abdominal obesity exhibits distinct effect on inflammatory and anti-inflammatory proteins in apparently healthy Japanese men. Cardiovasc Diabetol 2007;6: 27.

3 You T, Ryan AS, Nicklas BJ: The metabolic syndrome in obese postmenopausal women: relationship to body composition, visceral fat, and inflammation. J Clin Endocrinol Metab 2004;89:5517-5522.
4 Heinrich PC, Castell JV, Andus T: Interleukin- 6 and the acute phase response. Biochem J 1990;265:621-636.

5 Fontana L, Eagon JC, Trujillo ME, Scherer PE, Klein S: Visceral fat adipokine secretion is associated with systemic inflammation in obese humans. Diabetes 2007;56: 1010-1013.

6 Yudkin JS, Juhan-Vague I, Hawe E, Humphries SE, di Minno G, Margaglione M, Tremoli E, Kooistra T, Morange PE, Lundman P, Mohamed-Ali V, Hamsten A: Lowgrade inflammation may play a role in the etiology of the metabolic syndrome in patients with coronary heart disease: The HIFMECH study. Metabolism 2004;53:852857.

7 Wanner C, Metzger T: C-reactive protein a marker for all-cause and cardiovascular mortality in haemodialysis patients. Nephrol Dial Transplant 2002;17(Suppl 8):29-32, discussion 39-40.

$>8$ Krane V, Winkler K, Drechsler C, Lilienthal J, Marz W, Wanner C: Association of LDL cholesterol and inflammation with cardiovascular events and mortality in hemodialysis patients with type 2 diabetes mellitus. Am J Kidney Dis 2009;54:902-911. 
9 Zimmermann J, Herrlinger S, Pruy A, Metzger T, Wanner C: Inflammation enhances cardiovascular risk and mortality in hemodialysis patients. Kidney Int 1999;55: 648-658.

10 Gohda T, Gotoh H, Tanimoto M, Sato M, Io H, Kaneko K, Hamada C, Tomino Y: Relationship between abdominal fat accumulation and insulin resistance in hemodialysis patients. Hypertens Res 2008;31:83-88.

-11 Axelsson J, Rashid Qureshi A, Suliman ME, Honda H, Pecoits-Filho R, Heimburger O, Lindholm B, Cederholm T, Stenvinkel P: Truncal fat mass as a contributor to inflammation in end-stage renal disease. Am J Clin Nutr 2004;80:1222-1229.
12 Kaysen GA, Kotanko P, Zhu F, Sarkar SR, Heymsfield SB, Kuhlmann MK, Dwyer T Usvyat L, Havel P, Levin NW: Relationship between adiposity and cardiovascular risk factors in prevalent hemodialysis patients. J Ren Nutr 2009;19:357-364

13 Berg AH, Lin Y, Lisanti MP, Scherer PE: Adipocyte differentiation induces dynamic changes in NF-kappaB expression and activity. Am J Physiol Endocrinol Metab 2004; 287:E1178-E1188.

14 Trayhurn P, Wood IS: Adipokines: inflammation and the pleiotropic role of white adipose tissue. Br J Nutr 2004;92:347-355.
5 Kalantar-Zadeh K, Kopple JD, Kilpatrick RD, McAllister CJ, Shinaberger CS, Gjertson DW, Greenland S: Association of morbid obesity and weight change over time with cardiovascular survival in hemodialysis population. Am J Kidney Dis 2005;46:489500.

16 Kalantar-Zadeh K: Causes and consequences of the reverse epidemiology of body mass index in dialysis patients. J Ren Nutr 2005; 15:142-147.

17 Stenvinkel P, Alvestrand A: Inflammation in end-stage renal disease: sources, consequences, and therapy. Semin Dial 2002;15: 329-337. 